\title{
SOME APPLICATIONS OF THE IMAGING PROPORTIONAL CHAMBER
}

\author{
G.Charpak, W.Dominik, J.P.Fabre, J.Gaudaen, V.Peskov, F.Sauli and M.Suzuki
} CERN, Geneva, Switzerland

A.Breskin, R.Chechik and D.Sauvage

Weizmann Institute, Rehovot, Israel

ABSTRACT: Photons emitted by avalanches in gases can be detected with an image intensifier coupled to a solid state camera. Some vapors enhance the emission at wavelengths close to the visible. We describe progress made in the imaging of charged particles and of Cherenkov photons.

Proportional multiwire gaseous detectors with electronics readout are extremely powerful devices intrinsically capable of analyzing complex events. However, the high cost of the wire electronics restricts the granularity that can be achieved in practice. This is a severe limiting factor in cases where the event multiplicity is high or when very large detection volumes are required, as for example for the study of rare events (e.g. proton and double beta decay).

As an alternative to the electronics detection of charges produced in the avalanches, one can exploit the emitted photons to obtain optical images, in projection, of the ionized clusters in a gaseous detector $[1-3]$. The detector itself can be continuously active, and is intrinsically capable of high rates, just like conventional proportional chamber; the data acquisition rate is limited only by the recording or digitizing speed (about $20 \mathrm{~ms}$ per frame using standard television hardware). On the other hand, the granularity that can be obtained ( 250000 pixels with standard, cheap recorders) makes the optical device a very attractive one.

While it is well known that photons are copiously produced in electron avalanches, they are mostly emitted at short wavelenghts (in the vacuum ultraviolet domain) and are therefore very hard to detect, or are reabsorbed by the gas mixture when using organic quenchers. A systematic search in our group has allowed to identify several gas mixtures in which the photon yield at wavelenghts close to the visible is copious, and in which, moreover, high proportional gains can be obtained [3-5]. As it happens, two vapors commonly used in photosensitive chambers, TMAE [tetrakis(dimethylamine)ethylene], and TEA (triethylamine), when added to noble gases or their mixtures with methane, allow to obtain very large photon yield.

The emission spectrum of mixtures containing TEA is centered around $280 \mathrm{~nm}$ (see Fig. 1), while for TMAE mixtures the peak of emission is at $480 \mathrm{~nm}$ (Fig. 2)[5]. The shape of the spectra appears to be almost independent from the other components of the gas mixture, provided they are transparent in the region of emission. It is not understood at this point if the large photon yield is due to some process of internal wavelength shift between molecules, to direct scintillation of the photosensitive addition in the avalanches, or to both processes. The emision that we have measured in the mixture with TMAE coincides with the known fluorescence spectrum of the pure vapor [6].

We have measured the light yield of various gas mixtures containing TEA and TMAE under avalanching conditions, recording simultaneously the charge and the light detected on a photomultiplier mounted close to a parallel plate avalanche chamber with a $\mathrm{CaF}_{2}$ window. The results are collected in Fig.3, that provides the ratio of light and charge signals (in arbitrary units) as a function of charge gain for various nixtures. One can see that, for a given charge gain, the yield for TEA is more than an order of magnitude larger than for TMAE; one should not forget however that, because of the vapor pressure of the two compounds ( 70 torr and 0.5 torr, respectively, at room temperature) the concentration of scintillating molecules is very different in the two cases.

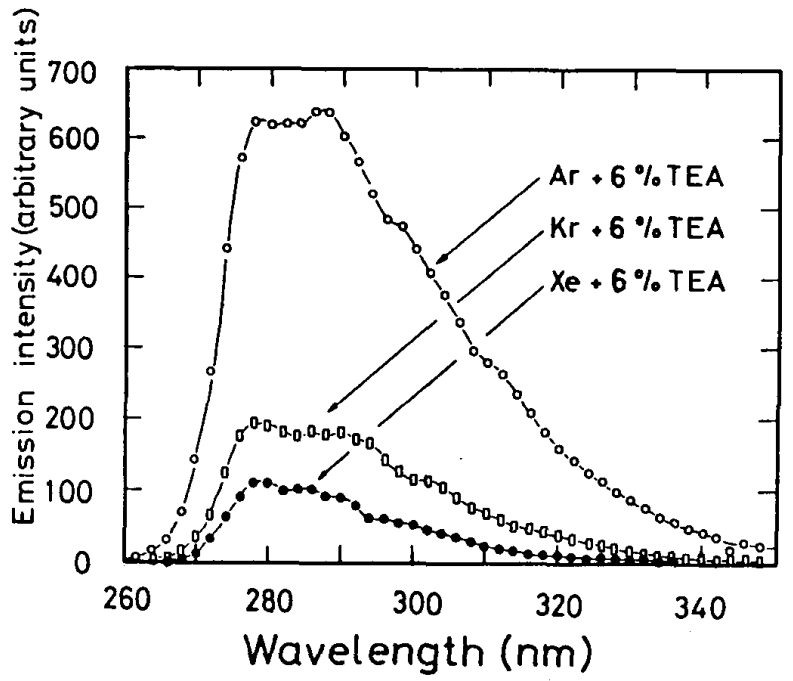

Fig. 1: Emission spectrum of TEA in mixtures with noble gases, measured under avalanching conditions in a proportional counter operated at atmospheric pressure.

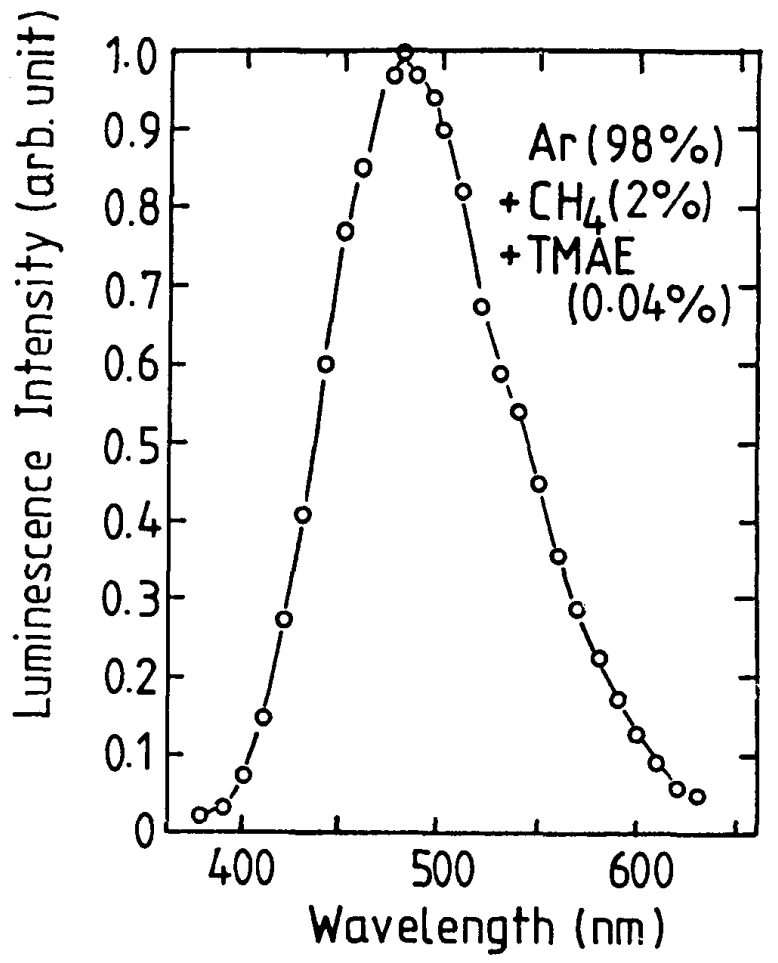

Fig. 2: Emission spectrum of TMAE in argonmethane. 


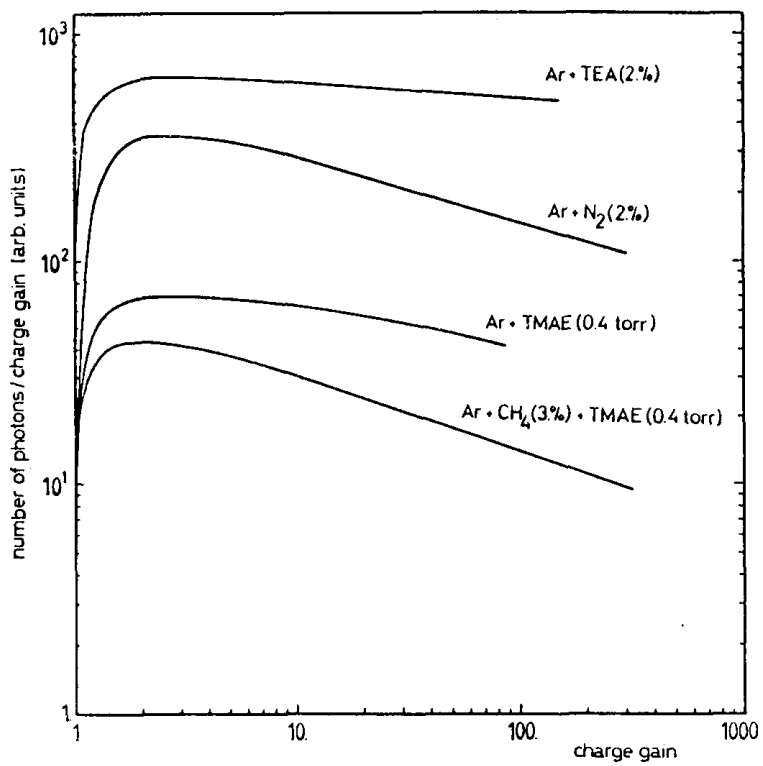

Fig. 3: Relative light yield, normalized to the charge gain, in various gas mixtures at atmospheric pressure under avalanche conditions.

The peak of TEA emission is at sufficiently long wavelengths to allow the use of thin polycarbonate windows (aclar [7], for example, has an absorption length of $200 \mu \mathrm{m}$ at $300 \mathrm{~nm}$ and a cutoff at $220 \mathrm{~nm}$ ); the major limitation comes however from the need of employing an optical system efficient at thes: wavelengths. In particular, one needs an objective made of UV grade quartz (expensive or limited in aperture), and a multialcali photocathode for the front end of the image intensifier, in general rather noisy. It is obviously more convenient to detect the longer wavelenghts of the TMAE emission, but the smaller light yield implies the use of larger chamber gains (such as those that can be obtained for example with multistep chambers or operating at low pressures, see below).

The detector that we have used for imaging charged tracks in projection is illustrated in Fig. 4. It consists in a large volume of drift followed by a multiplying element; the light emitted is detected with an image intensifier coupled to a standard television or to a solid state camera. The detector itself can be continuously active, or gated if a selection is desired (for example in a high radiation environment).

While the first images were obtained using a MWPC as amplifier, nnuch better results in terms of light yield and tracking resolution have been obtained using as light emitter a simple parallel plate avalanche chamber, five to ten $\mathrm{mm}$ thick. This is mostly due to the higher light emission efficiency at low electric fields (given the charge gain), see Fig. 3. Indeed, in a MWPC all the charge gain is produced in a short, very high field region, while in a parallel plate chamber the same gain is obtained multiplying the electrons over a long region of moderate field. An additional effect could be due in the parall plate device to the transverse avalanche spread that chliterates the shadowing effect of the wires in the anode mesh (as compared to what happens in a multiwire chamber). Fig. 5 shows some examples of tracks recorded in a minimum ionizing particles beam with the imaging chamber, operated with argon-methane-TEA.

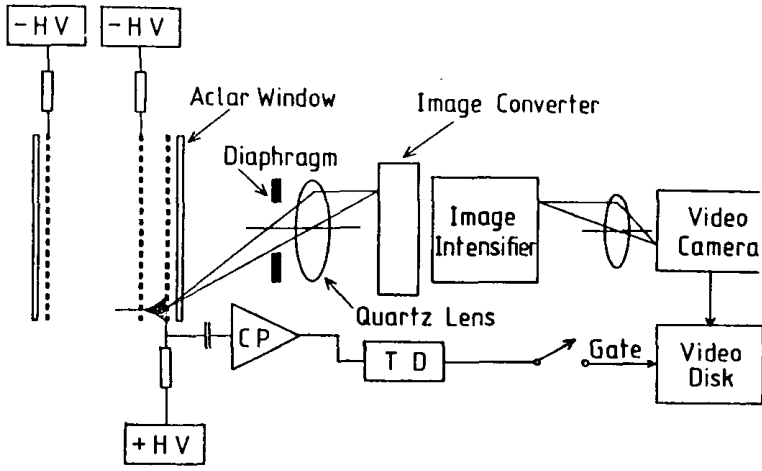

Fig. 4: Schematics and principle of operation of the imaging chamber.

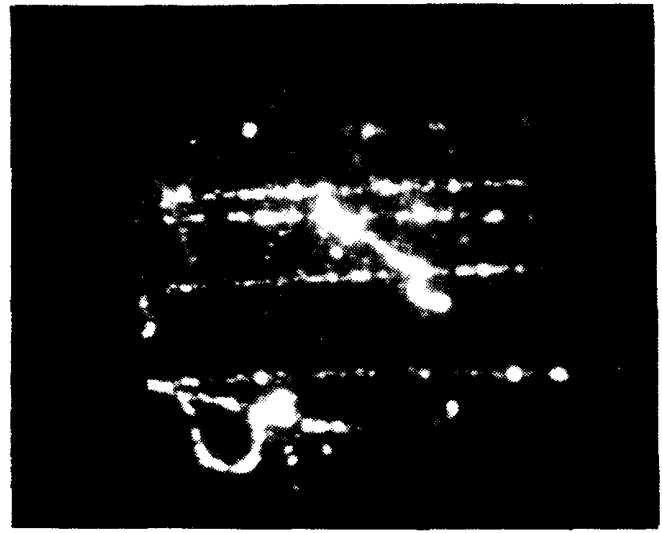

Fig. 5: Example of tracks recorded in a particle beam with the imaging chamber. The real size of each image is about $6 \times 6 \mathrm{~cm}^{2}$

The light emission is proportional to the energy loss in a large dynamic range, although the telcvision recording medium and the photographic reproduction largely deteriorate the response. For this reason we are now using a solid state camera and a frame digitizer, that allows to record images in real time with a computer. An example of digitized image, recorded with the imaging chamber in the detection of alpha particles of around $5 \mathrm{MeV}$, is shown in Fig. 6; the shade of the pixels (originally in color) represents the relative intensity of the light recorded, and an integration of the light intensity along each track very much reproduces the typical Bragg energy loss distribution.

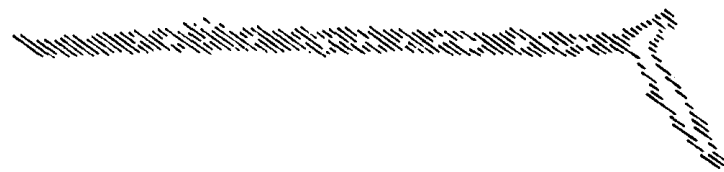

Fig. 6: An alpha particle interacting in the drift volume of the imaging chamber; the real track length is about $10 \mathrm{~cm}$ (gas filling: helium-TEA). Images were recorded with a CCD camera and digitized for analysis. 
Another interesting application of the imaging chamber is for the detection of photons emitted by Cherenkov effect in a radiator, and reflected in a circular image on the focal plane of a spherical mirror (the so-called Cherenkov ring imaging or $\mathrm{RICH}$ technique). Indeed, the vapors producing the largest photon yield are photosensitive and are used in conventional electronic $\mathrm{RICH}$ detectors based on multiwire proportional chambers.

While it is possible with conventional chambers to attain the large gains necessary for electronic or optical detection of avalanches in mixtures containing TEA, this is not so easy when using TMAE because of its very low vapor pressure. Ultraviolet photons emitted in the avalanches have a long absorption path in the gas, several $\mathrm{cm}$ or so, and can generate spurious secondary electrons in a number that can exceed the original number of photoelectrons. It was shown some time ago that a multistep proportional chamber allows to operate at very high gains in TMAE without detectable photon feedback [8]. Further work using a low-pressure multistep chamber has shown that even larger effective multiplication factors can be obtained (up to $10^{7}$ or so) without feedback problems [9]; moreover, a low pressure device is well suited for the use in experiments where one expects a large flux of charged particles to accompany the photons to be detected. We have realized a RICH imager as shown in Fig. 7 [10]. It consists in a gas radiator $1.7 \mathrm{~m}$ long, containing methane at atmospheric pressure, separated from the detector by a $\mathrm{CaF}_{2}$ window; photons emitted by fast charged particles in the radiator are reflected on the focal plane (the plane of detection) by a spherical mirror at one end of the radiator. The detector itself is a low-pressure multistep gated chamber filled with a gas mixture containing TMAE as photosensitive vapor, optimized for its light emission. An example of ring recorded in a minimum ionizing particles beam is shown in Fig. 8; in average, ten photons are deterted per event, as expected from the design parameters, proving that the efficiency of the optical imager is close to $100 \%$. The detected clusters are rather broad, due to diffusion and to the large transverse size of the avalanche, increased by the multistep mechanism at low pressures. This is obvicusly a limiting factor for two-clusters resolution; it could be largely improved operating the chamber at atmospheric pressure, but in this case, to avoid the problems connected with direct ionization of the gas by charged particles, one would have to reduce the detector thickness heating the device to operate with higher TMAE vapor pressures.

The position of each photon is computed from a twodimensional center of gravity of the detected cluster, and after a fit to a circle the dispersion of each measurement can be estimated. This is shown in Fig.9, that provides the distribution of the distances of each cluster from the fitted center of the circle; it has a standard deviation of $2.2 \mathrm{~mm}$.

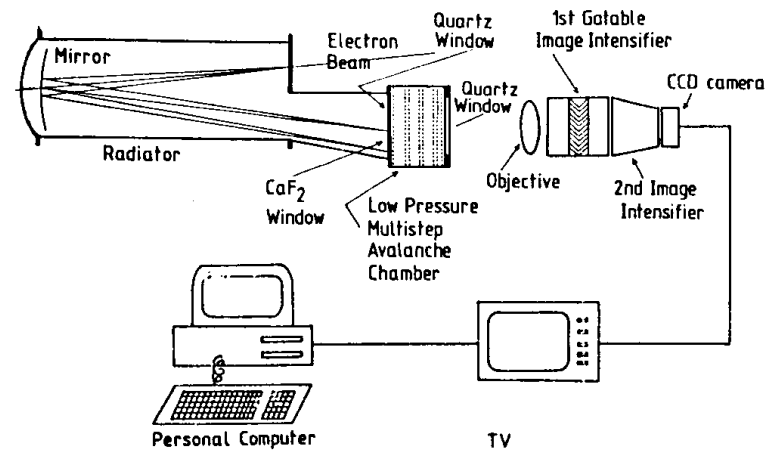

Fig. 7: Schematics of the imaging Cherenkov ring chamber.
3941 MAX: 92 AUG: 4514 POINTS 10 FIT: 10 SIGMA: 6.8

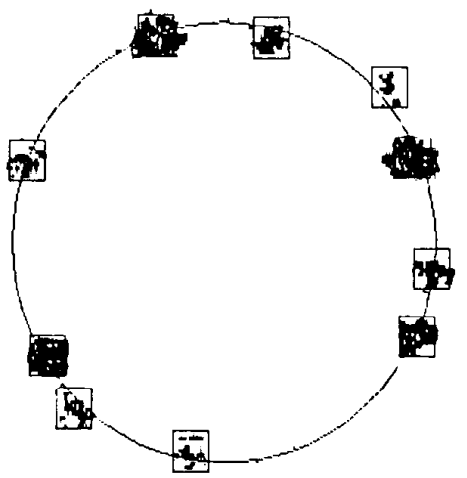

Fig. 8: An event recorded with the imaging Cherenkov ring chamber. Each cluster corresponds to the light emitted by avalanches produced by a detected photon; the result of the fit to a circle is also shown.

CLUSTER US R

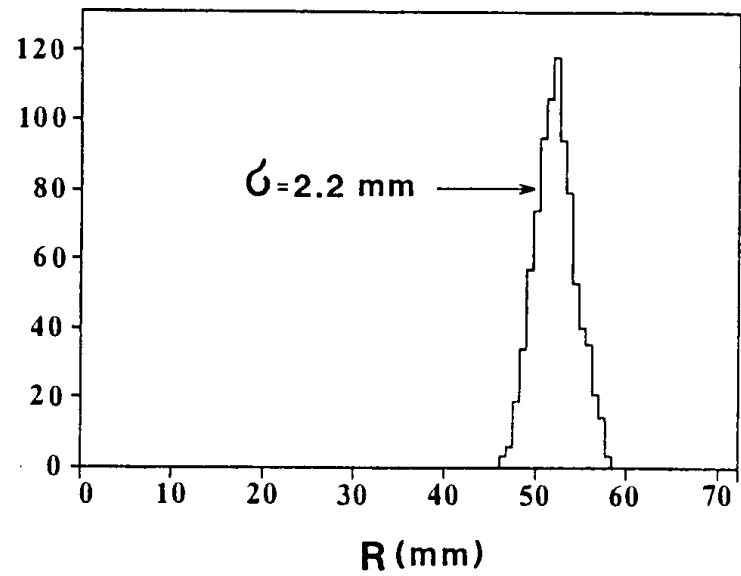

Fig. 9: Distribution of the center of gravity of the measured single-photon clusters; the rms of the distribution is $2.2 \mathrm{~mm}$.

We foresee application of this technique for Cherenkov Ring Imaging of heavy ions, where the large photon yield prevents any electronics system to be usable, and in astrophysics, for example in the imaging of the photons emitted in electromagnetic showers generated in a thick crystal radiator [11].

It was reported some time ago [12] that under suitable conditions one could deposit a layer of liquid TMAE on the cathode surface of a chamber; since one expects a lower photoionization threshold in the liquid phase $(4.2 \mathrm{eV}$ as compared to $5.4 \mathrm{eV}$ for the vapor), a substantial gain should be obtained for example in the detection of the fast scintillation component of $\mathrm{BaF}_{2}$. The value of the quantum efficiency for the condensed liquid remained however rather controversial [13]. In a parallel research on photosensitive detectors, and in collaboration with other authors, some of us have investigated the properties of condensed photocathodes chambers using a small size proportional counter with removable cathode, mounted on a monochromator for spectral analysis of the quantum yield [14]; here we report only some major observations. 
Fig. 10 shows the enhancement in quantum efficiency at long wavelengths obtained when simply exposing various metals to TMAE vapors, without any thermal gradient to provoke condensation; notice that the photoionization threshold of TMAE vapor is around $220 \mathrm{~nm}$. The enhancement persists when repeatedly evacuating the chamber, and is still observed after days of flushing with neutral gases; it disappears after thermal or mechanical treatment of the cathode, and is obviously due to a layer of adsorbed molecules on the metal surface. Although the absolute quantum efficiency is small, one can suspect the effect to be responsible of various incuinsistencies in measurements having to do with TMAE.

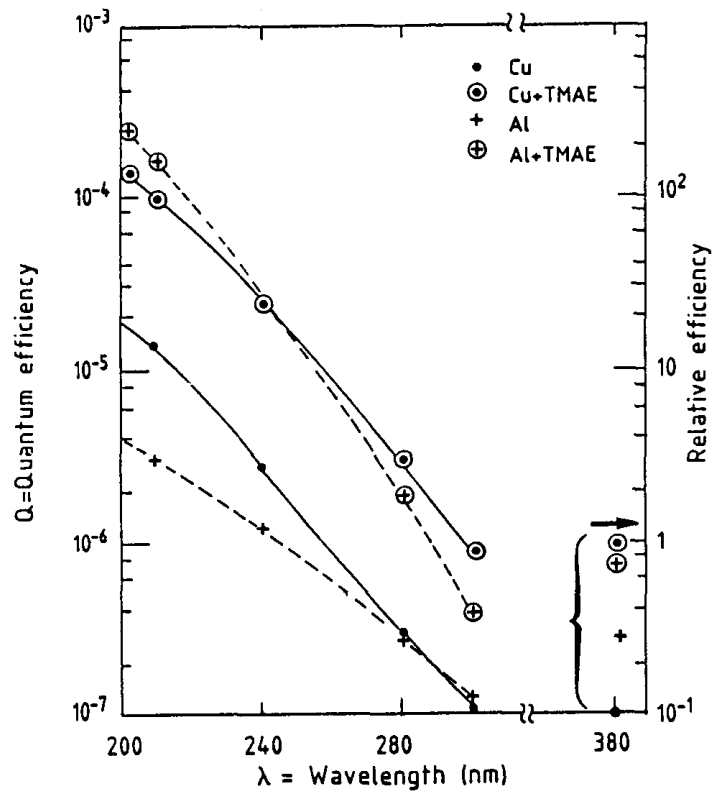

Fig. 10: Quantum efficiency as a function of wavelength measured in a proportional counter with metal cathodes before (lower pair of curves) and after exposure to TMAE at room temperature.

The quantum efficiency measured with a thick liquid layer of TMAE, obtained by condensation cooling the cathode body, is given in Fig.11 (curve 2). It obviously extends to much longer wavelengths than the vapor, but the absolute value is still rather low $\left(10^{-3}\right.$ at $\left.240 \mathrm{~nm}\right)$.

It is known that the photoionization threshold of TMAE in organic solutions is lower than in the vapor phase [6]. Recently, it has been verified that the same property holds for solutions in room - temperature liquids that have very long free electron lifetime, such as tetra-methyl-pentane (TMP), tetra-methyl-silane (TMS) and neopentane [15]. We have measured the quantum efficiencies of various composite liquid photocathodes; some results are collected in Fig. 11. The measurements correspond, respectively, to TMAE solutions in TMP (curve 4), in TMS (curve 5), in neopentane condensed at $0^{\circ} \mathrm{C}$ (curve 6) and at $-20^{\circ} \mathrm{C}$ (curve 7). In al caies, the proportional chamber was operated with $\mathrm{CH}_{4}$ at atmospheric pressure as gas filling. As one can see, the enhancement is rather substantial, especially for the last measurement; the quantum efficiency does not reach the high values anticipated for the solutions, but of course in our experimental setup one does not only re- quires a photoclectron to be produced in the liquid, but also to be emitted in the gas of the counter and detected. There are indications that a higher field at the cathode surface, which we were not able to attain with the present setup, should largely increase the yield. If this is experimentally confirmed, one can get close to the possibility of building in a rather simple way position-sensitive photon gaseous detectors of very large surfaces.

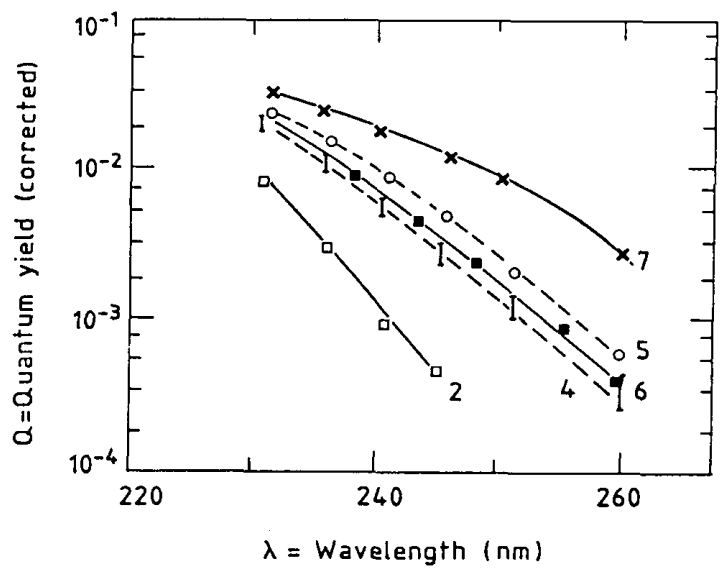

Fig. 11: Quantum efficiency of various liquids condensed on the cathode of a proportional counter. The meaurements are shown for pure TMAE (curve 2), TMAE dissolved in TMP (curve 4), TMAE in TMS (curve 5), TMAE in neopentane condensed at $0{ }^{\circ} \mathrm{C}$ (curve 6) and at $-20^{\circ} \mathrm{C}$.

\section{References}

R.S.Gilmore, T.K.Gooch, W.L.Kwan, I.C.McArthur, J.Malos, J.P.Melot, R.J.Tapper and R.J.Whyley, Nucl.Instr.Methods 206 (1983) 189.

[2] D.M.Potter, Nucl.Instr.Methods 228 (1984) 56.

[3] G.Charpak, J.P.Fabre, F.Sauli, M.Suzuki and W.Dominik, Nucl.Instr.Methods A258 (1987) 177.

[4] M.Suzuki, P.Strok, F.Sauli and G.Charpak, Nucl.Instr.Methods A254 (1987) 556.

[5] G.Charpak, J.P.Fabre, J.Gaudaen, F.Sauli, M.Suzuki and W.Dominik, Further studies of the light emission by continuous avalanche chambers (in preparation at CERN, 1987)

Y.Nakata, M.Ozaki and J.Phys.Chem. 76 (1972) 2105.

[7] Aclar $22 \mathrm{~A}$, polycloro-trifluoruro ethylene.

[8] G.Charpak and F.Sauli, Nucl.Instr.Methods 225 (1984) 627.

[9] A.Breskin and R.Chechik, Nucl.Instr.Methods A252 (1986) 488 .

[10] A.Breskin, R.Chechik, Z.Fraenkel, D.Sauvage, V.Steiner, I.Tserruya, G.Charpak, W.Dominik, J.P.Fabre, J.Gaudaen, F.Sauli, M.Suzuki, P.Glässel, H.Ries, .Shön and H.Spacht, WIS-87/68. Pres. at the London Conf. on Position Sensitive Detectors (London, 7-11 Sept. 1987)

[11] I.Giomataris and G.Charpak, 20th Intern. Cosmic Ray Conf., Moscow Aug.1987.

[12] D.Anderson, Phys.Letters 118B (1982) 230

[13] J.C.Michau, B.Pichard, J.P.Soirat, M.Spiro and D.Vignaud, Nucl.Instr. Methods A244 (1986) 565

[14] V.Peskov, G.Charpak, Ph.Mine, F.Sauli, D.Scigoki, J.Seguinot, W.F. Schmidt and T.Ypsilantis, Search on Liquid Photocathodes (In preparation at CERN)

[15] R.A.Holroyd et al, J.Phys.Chem.89 (1985)4244 